ORIGINAL ARTICLE

\title{
The 'Quiet Eye' and motor performance in basketball free throw shooting
}

\author{
Ece Ayaz Kanat ${ }^{1 \mathrm{ABCDE}}$, Deniz Şimşek ${ }^{2 \mathrm{ABCDE}}$ \\ ${ }^{1}$ Eskişehir Anadolu University, Turkey \\ ${ }^{2}$ Eskişehir Technical University, Turkey
}

Authors' Contribution: A - Study design; B - Data collection; C - Statistical analysis; D - Manuscript Preparation; E - Funds Collection.

\begin{abstract}
Background This study investigates expert and amateur basketball players' visual tracking strategies accuracy and and Study Aim inaccuracy free throws.

Material and A total of 22 university student, 11 of whom were experts ( $n=5$ females, $n=6$ males) and 11 amateurs Methods ( $\quad n=5$ females, $n=6$ males), without visual impairment, participated the study. Each athlete performed a total of 10 throws. The normal distribution of data recorded in both accurate and inaccurate throws was checked with the Shapiro-Wilk test. All of the data received on accurate and inaccurate throws were analyzed by independent samples t-test.

Results $\quad$ Expert basketball players were recorded to have an average of $78 \%$ accurate throws out of 10 , and as for amateur basketball players, they were recorded to have $34 \%$. During accurate throws, the quiet eye durations of expert basketball players were identified as $\sim 886 \mathrm{~ms}$, and quiet eye durations during inaccurate throws were as $\sim 570 \mathrm{~ms}$. During accurate throws, the quiet eye durations of amateur basketball players were identified as $\sim 612 \mathrm{~ms}$, and quiet eye durations during inaccurate throws were as $\sim 388 \mathrm{~ms}$. The study's findings reveal that expert basketball players develop statistically longer quiet eye and fixation durations than amateur basketball players.

Conclusions: The study's findings have supported the phenomenon of longer quiet eye durations during expert athletes' specific motor skills. The knowledge obtained from the study's findings is thought to have the utmost significance in identifying the quiet eye durations of expert basketball players and teaching their visual strategies to amateur level athletes to improve their performance.

Keywords: eye strategies, quiet eye, free throw, basketball, motor control
\end{abstract}

\section{Introduction}

The road to sports expertise is a difficult process explored in various sports disciplines [1]. Studies show that skillful athletes develop superior perceptualcognitive skills, referring to the ability to find and identify visual information [2] in the environment for selecting and executing actions, in addition to physiological, emotional, and technical skills [3]. In this context, talented athletes were stated to anticipate better the actions of their opponents [4-6], and the most important process underlying this situation was the improved decision-making skills of expert athletes [7, 8] and motor performances [9-11]. Visual strategies are important to achieve high scores, especially during motor skills that require shooting or aiming at the target. Among those sports branches, basketball free throws are vitally important to win the game. [12-15]. In recent years, numerous studies conducted on basketball players provide eye movement records [16-19]. The studies revealed that expert basketball players or expert athletes performing other motor tasks developed longer quiet eye duration $[11,15,20]$.

The quiet eye is identified as a minimum $100 \mathrm{~ms}$ final fixation at a visual angle of 3 degrees (or less) to a specific region or object within the task area. The quiet (c) Ece Ayaz Kanat, Deniz Şimşek, 2021 doi:10.15561/20755279.2021.0205 eye's start occurs before the critical movement phase, and the end of the quiet eye occurs as the gaze movement goes beyond the visual angle of 3 degrees or $100 \mathrm{~ms}$. [21-24]. Examining the mechanism underlying quiet eye: it has been regarded that the long fixation time on the specific point before the final movement is necessary to organize the neural connections underlying skill control [25]. This is particularly related to the paradoxical finding of increased quiet eye duration related to increased motor expertise; Mann et al. [26] called it "the productivity paradox," though.

On the one hand, based on motor expertise, this paradox is overall based on the observation characterized by behavior economics and the "automation" underlying various control processes [27]. The quiet eye is stated to reflect the duration required for motor preparation to ensure the required movement parameters just before performing a particular movement and the limbs' timingcoordination in various studies [28-30]. In this way, longer quiet eye periods extend this critical preparation period's duration and turn into a higher chance of motor performance [3]. There is evidence that in various sports such as shooting, compared to novice athletes, expert athletes exhibit a visual pattern of less fixation for more prolonged periods [29, 31-33]. The ability to select appropriate visual stimuli and process them effectively, 
particularly during motor tasks involving shooting and aiming, maintains the optimum level of concentration for an accurate performance as well [28]. While the quiet eye duration varies depending on specific task demands, studies reveal that expert athletes exhibit longer quiet eye durations than novices [28, 29]. Besides, studies have found out that longer quiet eye durations correlate with accurate results [30, 34]. Based on the studies conducted, it is considered that determining the quiet eye durations of expert basketball players and teaching those visual strategies exhibited by expert athletes to amateurintermediate level athletes will be of great importance. The study aims to investigate the visual control strategies that expert and amateur basketball players exhibit during free throws. The study hypothesizes that expert basketball players will exhibit a higher percentage of accuracy and attention control (longer quiet eye durations) than amateur basketball players.

\section{Material and Methods}

\section{Participants}

Twenty-two basketball players participated in the study (expert: $\mathrm{n}=11,20,71 \pm 1,57$ age; $1,81 \pm 0,09 \mathrm{~cm}$; $75,1 \pm 4.0 \mathrm{~kg} ; 9,9 \pm 2,92$ year; Amateur: $\mathrm{n}=11,18,75 \pm 1.06$ age; $180,45 \pm 6,85 \mathrm{~cm} ; 67,75 \pm 15,76 \mathrm{~kg} ; 1,00 \pm 0,00$ year). This study was conducted in October during the 2017/2018 season. The groups participating in the study consisted of amateur and expert athletes. All expert athletes were selected among those who played in the same league in the same season. Players who use the dominant hand right hand participated in the study. Each participant was asked to sign a voluntary consent form. Eskişehir Osmangazi University clinical research ethics committee approved the study's ethical permissions with document number $80558721 / 32$. The demographic information about the athletes is given in Table 1.

Research Design

Tobii Pro Glasses 2 (Tobii Pro Glasses 2, Stockholm, Sweden) brand eye tracking device was used to examine the participants' visual search behavior during a free throw. During the free-throw, visual search behaviors were recorded online via Tobii Glasses Controller (Glasses controller Software, Stockholm, Sweden) software specific to the eye tracker. Measurements were recorded in daylight and at a recording rate of $100 \mathrm{~Hz}$. The system's working principle depends on recording the perspective on a video image of the binocular corneal reflection to an integrated camera, measuring the pupil's related position and the corneal reflection. The device was placed on the face most comfortably using special nose pads peculiar to each participant's facial structure. After placing the eye tracker on the face, the calibration process was repeated for each throw of the participants to adapt it with the Tobii Glasses Controller software (Glasses controller Software, Stockholm, Sweden) the data online.

The participants were allowed to warm up for 20 minutes according to a standard warm-up protocol before all measurements. How the free throw should be done technically was shown by the trainer before the test (fig. 1). Free throws were made according to the game rules. Participants were told to throw the ball into the basket within 5 seconds after the ball was thrown using

Table 1. Demographic information about basketball players and their free throw percentage accuracy values from the previous season.

\begin{tabular}{llllll}
\hline Participants & $\begin{array}{l}\text { Age (year) } \\
\text { Mean } \pm \mathbf{s d}\end{array}$ & $\begin{array}{l}\text { Height }(\mathbf{c m}) \\
\text { Mean } \pm \mathbf{~ s d}\end{array}$ & $\begin{array}{l}\text { Weight (kg) } \\
\text { Mean } \pm \mathbf{s d}\end{array}$ & $\begin{array}{l}\text { Shooting } \\
\text { percentage } \\
(\%)\end{array}$ & $\begin{array}{l}\text { Experience (year) } \\
\text { Mean } \pm \mathbf{s d}\end{array}$ \\
\hline $\begin{array}{l}\text { Expert } \\
\text { Group(n=11) }\end{array}$ & $20,82 \pm 3,02$ & $1,82 \pm 0,08$ & $76,00 \pm 13,82$ & 78 & $10,09 \pm 3,70$ \\
$\begin{array}{l}\text { Amateur } \\
\text { Group(n=11) }\end{array}$ & $18,75 \pm 1,21$ & $180,00 \pm 7,13$ & $67,50 \pm 13,31$ & 34 & $1,00 \pm 0$ \\
\hline
\end{tabular}

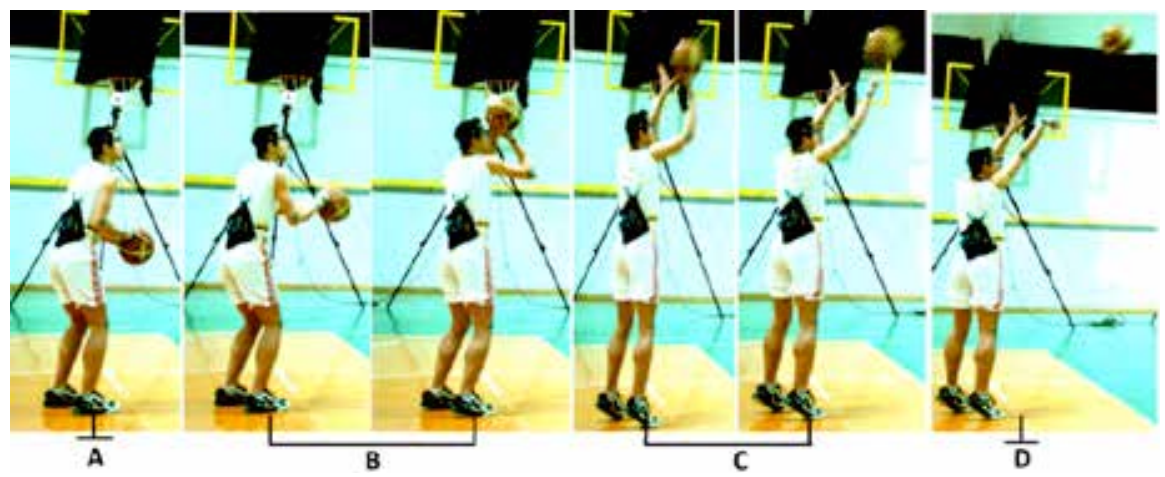

Fig. 1. Phases of free-throw A- Preparation phase: It is the process in which visual information is received before starting the action; B- Acceleration: It is the process in which the shooting movement begins, phased as its beginning and end; C-Shooting: the moment of the shooting and the process of handing the ball; D- Flight: It is the process that starts with the last wrist movement after the ball is released and ends with the release [35]. 
the bounce pass technique. Participants were allowed to throw 15 trial shots to get used to the test protocol. In this way, the athletes' adaptation to the glasses was provided, and calibration adjustments were made. Participants shot ten free throws from the free-throw line following the protocol. A break of 3 minutes was given between each throw. Each throw was recorded as accurate or inaccurate, and also measurements were made between 09:00 and 17:30 in Anadolu University Sports Hall on non-training days. The measurement period lasted 120 minutes for each participant. Participants were restricted to intake of caffeine and similar stimulants before measurements.

\section{Data analysis}

Expert and amateur participants shot ten free throws during the measurement. Free throws were grouped as accurate and inaccurate shots, and their averages and percentages were identified. The raw data recorded by the eye tracker was analyzed with Tobii Pro Lab software, an analysis system specific to the eye tracker. Having the raw data transferred to the software, they were examined in terms of interest areas determined for each record. For expert and amateur players participating in the study, 220 individual records were obtained from 220 throws. Each of the 220 records was analyzed individually in the same way under the determined standards. The recording was started when the ball was released from the passing coach and ended when the ball left the athlete's fingertips and touched the basket. The passing phase started with the video's first frame showing the ball's release from the passing coach and ended with the phase before the ball first touched the participant's hands. Shooting arm flexion was determined to begin when the elbow angle decreased as the ball was lifted from the body's midline and above the head. Arm extension began with the first frame showing the elbow angle until the ball left the fingertips; the shooting phase lasted until the ball was released. It was regarded as necessary for the neuromotor skill to be defined for the participants to see if it was accurate, although they had no control over the shoot after the throw. The motor movement phases were determined by the camera recording taken from the sagittal with an external camera. Movement phases were determined kinematically as preparation phase, acceleration phase, shooting, and flight phase. After determining the movement phases, the fixations starting from 0 and the last and longest gaze before the shooting moment (quiet eye duration) were analyzed. For the quiet eye, which determined the preshooting neural programming process, a fixation occurred when the participant's gaze was fixed in a position for a minimum of $100 \mathrm{~ms}$ ( 3 video frames) and was analyzed with a probability of deviation of $0.25^{\circ}$ of the visual angle (the width of the cursor shown on the hoop in Fig. 2). The quiet eye was defined as the last fixation before starting the movement [22, 23, 36, 37].

Areas of interest were determined as hoop and backboard. While determining the fixations on the hoop and backboard, the quiet eye duration just before the release (the moment of release of the ball) phase was considered.

\section{Statistical Analysis}

Each player shot ten free throws in total. The data recorded inaccurate and inaccurate shots were processed by the Tobii Pro Lab software system and exported as excel files. SPSS 22.0 package program was used for data analysis. The normal distribution of the data recorded accurate and inaccurate shoots were checked with the Shapiro-Wilk test.

\section{Results}

Statistically significant $(\mathrm{p}<0.05)$ differences were observed between the amateur and expert groups participating in the study. Firstly, examining the participant groups' average free throws, It was recorded that accurate free throws of the expert players were $78 \%$ and the inaccurate ones were $22 \%$, while the accurate free throws of the amateur athletes were $34 \%$ and the inaccurate ones were $66 \%$. The percentage of free throws of the players is shown in figure 3 and figure 4 .

Examining the quiet eye duration of the accurate throws, the quiet eye duration of expert basketball players during accurate throws was identified to be $\sim 886 \mathrm{~ms}$, while the quiet eye duration of amateur basketball players

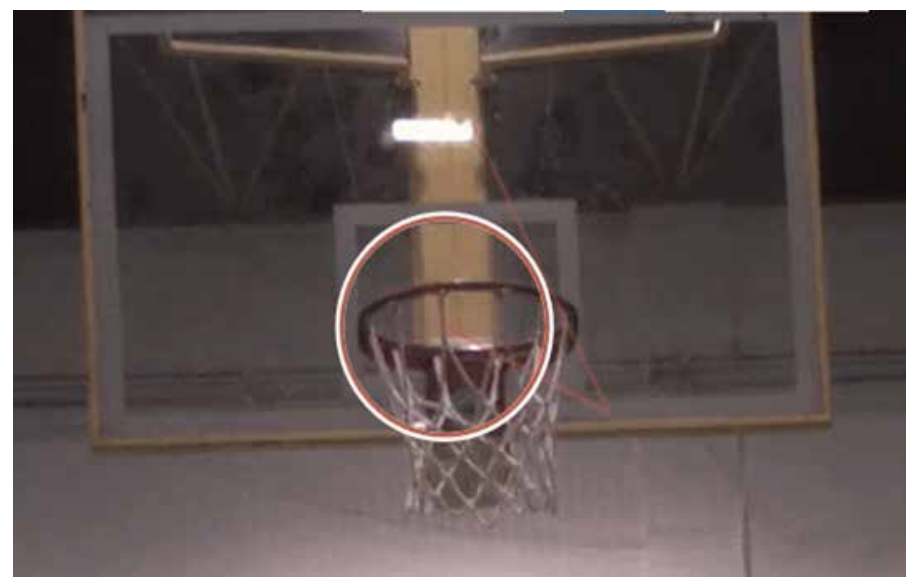

Fig. 2. The Moment of Fixation on the Hoop Taken from the Eye Tracking System 
during accurate throws was $\sim 612 \mathrm{~ms}$. As for the quiet eye duration of inaccurate shoots, the quiet eye period of expert basketball players during the shooting was $\sim 570 \mathrm{~ms}$, and the quiet eye duration of amateur basketball players was $\sim 388 \mathrm{~ms}$. The quiet eye duration for inaccurate free throws is shown in milliseconds in figure 5.

Examining the average fixation time spent on the hoop, which is one of the areas of interest during accurate shots, expert basketball players' fixation time was found as $\sim 1.09 \mathrm{~ms}$, and amateur basketball players' fixation time was $\sim 0.51 \mathrm{~ms}$. When the average fixation time spent on the backboard, which is one of the areas of interest determined during accurate shots, expert basketball players' fixation time was determined as $\sim 0,04 \mathrm{~ms}$, and the fixation time of amateur basketball players was $\sim 0,30 \mathrm{~ms}$. Examining the average fixation time spent on the hoop, which is one of the areas of interest during inaccurate shots, expert basketball players' fixation time was found as $\sim 1,16 \mathrm{~ms}$, and amateur basketball players' fixation time was $\sim 0.53 \mathrm{~ms}$. When the average fixation time spent on the backboard, which is one of the areas of interest determined during inaccurate shots, expert basketball players' fixation time was determined as $\sim 0,15 \mathrm{~s}$, and the

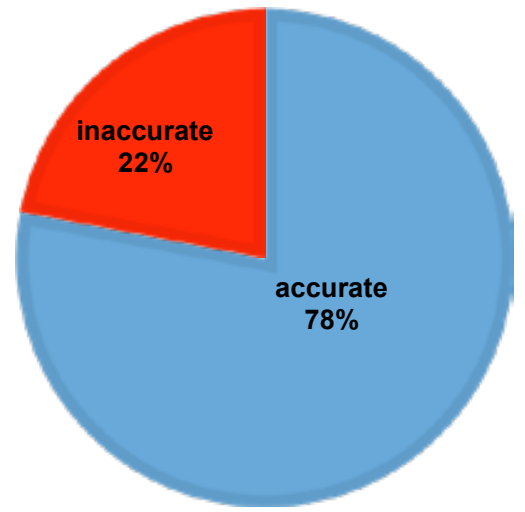

Fig. 3. Expert Group Free Throw Percentage fixation time of amateur basketball players was $\sim 0,82 \mathrm{~s}$. The average fixation time on the areas of interest during accurate and inaccurate free throws is shown in figure 6.

\section{Discussion}

Accurate free throws require accuracy, precision, concentration, shooting and gaze strategy, and mechanical efficiency $[24,25,38,39]$. Studies show that basketball's gaze strategy is the most significant variable that ensures accuracy in a free throw [40-42]. This study aimed to reveal expert and amateur basketball players' visual behavior strategies during the free throw.

The findings of this study confirm the research hypothesis. In other words, it reveals that a longer quiet eye duration characterizes the visual tracking strategies of expert athletes during both accurate and inaccurate free throws. Examining the results of the research in the literature investigating the relationship between the quiet eye period and experience level, it was seen that experts focus on the specified areas of interest for a longer period than non-experts $[34,43,44]$. Comparing the gaze of expert and intermediate shooters during free throws, Vickers [21] observed that expert shooters focused on the

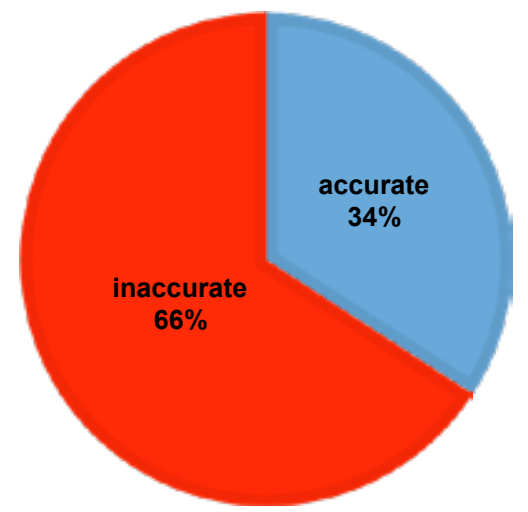

Fig. 4. Amateur Group Free Throw Percentage

\section{Quiet Eye Duration}

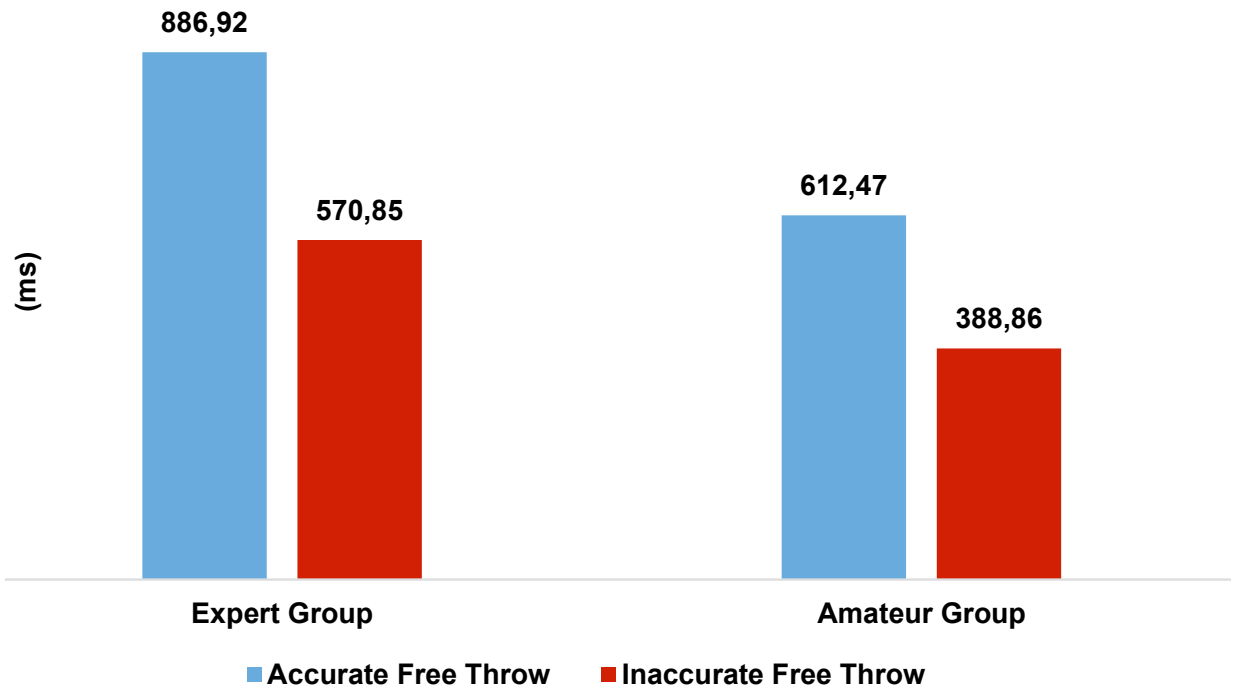

Fig. 5. Quiet eye duration of expert and amateur groups inaccurate and inaccurate free throws 


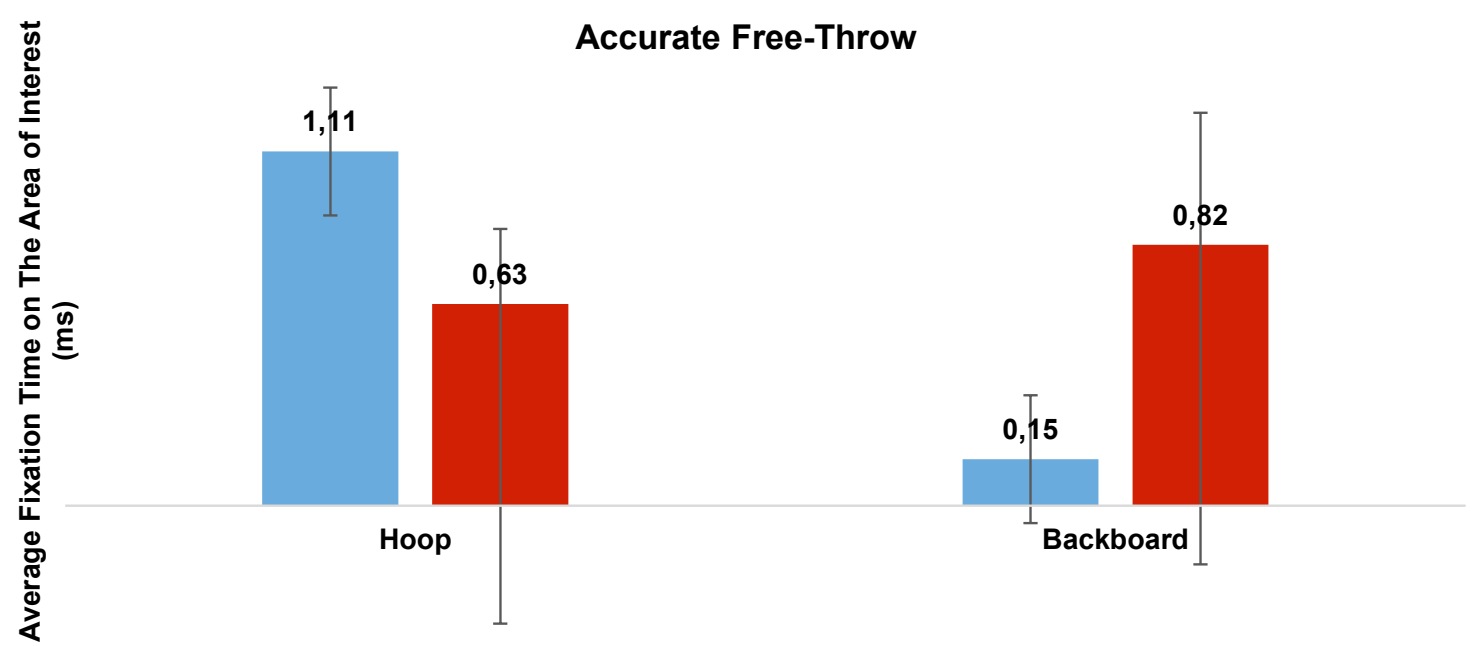

$\square$ Expert $\square$ Amateur

Inaccurate Free-Throw

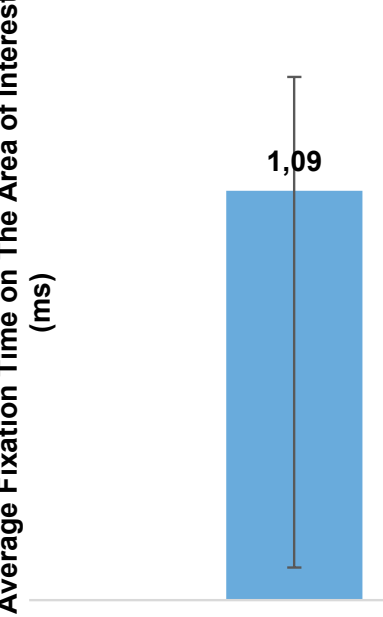

Hoop

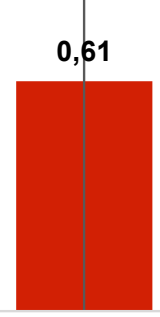

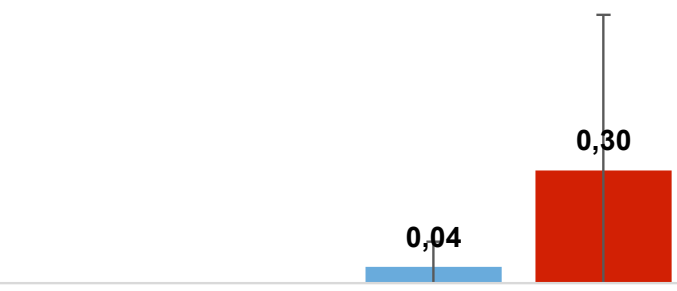

Backboard

Expert $\quad$ Amateur

Fig. 6. The average fixation duration on the areas of interest during accurate and inaccurate free throws

target for more than twice as long as intermediate shooters (972 vs. $357 \mathrm{~ms}$ ). In a similar study conducted by Steciuk and Zwierko [19], the total fixation duration of expert basketball players for accurate shoots was $916 \mathrm{~ms}$, and increased accuracy rates were positively associated with the prolongation of the average quiet eye duration. While Klostermann [45] compared the shooting accuracy as a function of the quiet eye duration, his study concluded that basketball players who displayed longer quiet eye duration had more accurate free throws. The research results of the current study are similar to the results of this mentioned research. It allows expert athletes to make detailed programs for the required shooting movements for long fixation durations towards the determined target (bucket, target) [46]. In other words, a longer quiet eye duration can be said to enable both the pre-planning of the movement and the realization of the next action and the use of visual knowledge while performing it [44]. Williams et al. [47] stated that long visual fixings were required to pre-program various motion parameters such as direction, force, speed, timing, and limb coordination.

Moreover, it was stated that long fixations to the target increased performance and the most critical reason underlying this was that experts (expert athletes) used that time for psychological and physiological regulation [47].

\section{Conclusion}

The findings of this study are consistent with other researchers who believe that the length of quiet eye durations of expert basketballers is necessary to program the direction of free-throw movement, strength, and speed, as well as limb coordination and timing [43]. This is because expert basketball players had a quiet eye duration of $913 \mathrm{~ms}$, and they exhibited $78 \%$ accuracy in their shooting percentage, which supports this situation. As a result, it has been determined that the spatial perception process has cruciality on motor programming (detection of the target and programming of targeted motion). 


\section{Suggestions}

This research's findings are thought to be of great significance in determining the quiet eye durations of expert basketball players and teaching these visual strategies exhibited by experts to amateur ones. Visual tracking strategies of high-level basketball players can be identified, and these data can be used as training materials. By creating a training program, training programs can be optimized for each player.

\section{Acknowledgments}

Special thanks are also given to the Turkey basketball super league basketball player.

This study was presented as an oral presentation, "The Quiet Eye Duration and Motor Expertise: free throw in basketball," at 16th International Sports Sciences Congress. This work won the best research award at the 16th International Sports Science Congress.

\section{References}

1. Baker J, Farrow D. Routledge handbook of sport expertise. London: Routledge, Taylor \& Francis Group; 2017.

2. Farrow D, Baker J, MacMahon C. Developing sport expertise. Abingdon: Routledge; 2013. https://doi.org/10.4324/9780203119914

3. Broadbent D, Causer J, Williams A, Ford P. Perceptualcognitive skill training and its transfer to expert performance in the field: Future research directions. European Journal of Sport Science, 2014;15(4):322-331. https://doi.org/10.1080/17461391.2014.957727

4. Abernethy B, Russell D. The relationship between expertise and visual search strategy in a racquet sport. Human Movement Science, 1987;6(4):283-319. https://doi.org/10.1016/0167-9457(87)90001-7

5. Ward P, Williams A. Perceptual and Cognitive Skill Development in Soccer: The Multidimensional Nature of Expert Performance. Journal of Sport and Exercise Psychology, 2003;25(1):93-111. https://doi.org/10.1123/jsep.25.1.93

6. Williams M, Davids K, Burwitz L, Williams J. Cognitive Knowledge and Soccer Performance. Perceptual and Motor Skills, 1993;76(2):579-593. https://doi.org/10.2466/pms.1993.76.2.579

7. Hancock D, Ste-Marie D. Gaze behaviors and decision making accuracy of higher- and lower-level ice hockey referees. Psychology of Sport and Exercise, 2013;14(1):66-71. https://doi.org/10.1016/j.psychsport.2012.08.002

8. Starkes J. Cognitive issues in motor expertise. Amsterdam: North Holland; 1993.

9. Roca A, Ford P, McRobert A, Williams A. PerceptualCognitive Skills and Their Interaction as a Function of Task Constraints in Soccer. Journal of Sport and Exercise Psychology, 2013;35(2):144-155. https://doi.org/10.1123/jsep.35.2.144

10.Ward P, Williams A. Perceptual and Cognitive Skill Development in Soccer: The Multidimensional Nature of Expert Performance. Journal of Sport and Exercise Psychology, 2003;25(1):93-111. https://doi.org/10.1123/jsep.25.1.93

11. Mann D, Williams A, Ward P, Janelle C. PerceptualCognitive Expertise in Sport: A Meta-Analysis. Journal of Sport and Exercise Psychology, 2007;29(4):457-478. https://doi.org/10.1123/jsep.29.4.457

12.Causer J, Holmes P, Smith N, Williams A. Anxiety, movement kinematics, and visual attention in elitelevel performers. Emotion, 2011;11(3):595-602. https://doi.org/10.1037/a0023225

13.Oudejans R, Karamat R, Stolk M. Effects of Actions Preceding the Jump Shot on Gaze Behavior and Shooting Performance in Elite Female Basketball Players. International Journal of Sports Science \& Coaching, 2012;7(2):255-267. https://doi.org/10.1260/1747-9541.7.2.255
14.Sáez-Gallego N, Vila-Maldonado S, Abellán J, Contreras Jordán O. El comportamiento visual de bloqueadoras juveniles de voleibol y su relación con la precisión de su respuesta. Cuadernos de Psicología del Deporte, 2015;15(2):143-154. https://doi.org/10.4321/S1578-84232015000200016

15.Rienhoff R, Tirp J, Strauß B, Baker J, Schorer J. The 'Quiet Eye' and Motor Performance: A Systematic Review Based on Newell's ConstraintsLed Model. Sports Medicine, 2015;46(4):589-603. https://doi.org/10.1007/s40279-015-0442-4

16.Steciuk, H, Zwierko T. Gaze behavior in basketball shooting: preliminary investigations. Trends in Sport Sciences, 2015;22(2).

17.Fischer L, Rienhoff R, Tirp J, Baker J, Strauss B, Schorer J. Retention of Quiet Eye in Older Skilled Basketball Players. Journal of Motor Behavior, 2015;47(5):407-414. https://doi.org/10.1080/00222895.2014.1003780

18.Krzepota J, Stępiński M, Zwierko T. Gaze Control in One Versus One Defensive Situations in Soccer Players With Various Levels of Expertise. Perceptual and Motor Skills, 2016;123(3):769-783. https://doi.org/10.1177/0031512516664903

19.Klostermann A, Panchuk D, Farrow D. Perceptionaction coupling in complex game play: Exploring the quiet eye in contested basketball jump shots. Journal of Sports Sciences, 2017;36(9):1054-1060. https://doi.org/10.1080/02640414.2017.1355063

20.Lebeau J, Liu S, Sáenz-Moncaleano C, SanduveteChaves S, Chacón-Moscoso S, Becker B et al. Quiet Eye and Performance in Sport: A Meta-Analysis. Journal of Sport and Exercise Psychology, 2016;38(5):441-457. https://doi.org/10.1123/jsep.2015-0123

21.Vickers J. Control of Visual Attention during the Basketball Free Throw. The American Journal of Sports Medicine, 1996;24(6_suppl):S93-S97. https://doi.org/10.1177/036354659602406S25

22.Vickers J. The Quiet Eye: Origins, Controversies, and Future Directions. Kinesiology Review, 2016;5(2):119-128. $\mathrm{https://doi.org/10.1123/kr.2016-0005}$

23.Vine S, Wilson M. Quiet Eye Training: Effects on Learning and Performance Under Pressure. Journal of Applied Sport Psychology, 2010;22(4):361-376. https://doi.org/10.1080/10413200.2010.495106

24. Wilson M, Vine S, Wood G. The Influence of Anxiety on Visual AttentionalControlinBasketballFreeThrowShooting.Journal of Sport and Exercise Psychology, 2009;31(2):152-168. https://doi.org/10.1123/jsep.31.2.152

25.de Oliveira R, Oudejans R, Beek P. Gaze Behavior in Basketball Shooting. Research Quarterly for Exercise and Sport, 2008;79(3):399-404. https://doi.org/10.1080/02701367.2008.10599504

26.Mann D, Wright A, Janelle C. Quiet Eye: The efficiency paradox -comment on Vickers. Current 
Issues in Sport Science (CISS), 2016;2016(1) https://doi.org/10.15203/CISS_2016.111

27.Fitts P, Posner M. Human performance. Westport, Conn.: Greenwood Press; 1979.

28.Causer J, Bennett S, Holmes P, Janelle C, W1lliams A. QuietEye Duration and Gun Motion in Elite Shotgun Shooting. Medicine \& Science in Sports \& Exercise, 2010;42(8):1599-1608. https://doi.org/10.1249/MSS.0b013e3181d1b059

29.Lee S M, Kim S, Park S H, et al. Self-paced sport events under temporal constraints: visual search, quiet eye, expertise and constrained performance time in far aiming tasks. International Journal of Applied Sports Sciences, 2009;21(2).

30.Wilson M, Pearcy R. Visuomotor Control of Straight and Breaking Golf Putts. Perceptual and Motor Skills, 2009;109(2):555-562. https://doi.org/10.2466/pms.109.2.555-562

31.Vickers J. Visual control when aiming at a far target. Journal of Experimental Psychology: Human Perception and Performance, 1996;22(2):342-354. https://doi.org/10.1037/0096-1523.22.2.342

32.Cañal-Bruland R, Zhu F, der Kamp J, Masters R. Targetdirected visual attention is a prerequisite for action-specific perception. Acta Psychologica, 2011;136(3):285-289. https://doi.org/10.1016/j.actpsy.2010.12.001

33.Piras A, Lobietti R, Squatrito S. Response Time, Visual Search Strategy, and Anticipatory Skills in Volleyball Players. Journal of Ophthalmology, 2014;2014:1-10. https://doi.org/10.1155/2014/189268.

34.Williams A, Singer R, Frehlich S. Quiet Eye Duration, Expertise, and Task Complexity in Near and Far Aiming Tasks. Journal of Motor Behavior, 2002;34(2):197-207. https://doi.org/10.1080/00222890209601941

35. Watanabe T, Sato T, Igawa S. Accuracy of Skill Performance in the Basketball Free Throw Shooting. BIO Web of Conferences, 2011;1:00096. https://doi.org/10.1051/bioconf/20110100096

36.Vickers J. Perception, cognition, and decision training. Champaign, Ill: Human Kinetics; 2007.

37. Wilson M, Wood G, Vine S. Anxiety, Attentional Control, and Performance Impairment in Penalty Kicks. Journal of Sport and Exercise Psychology, 2009;31(6):761-775. https://doi.org/10.1123/jsep.31.6.761

38. Harle S, Vickers J. Training Quiet Eye Improves Accuracy in the Basketball Free Throw. The
Sport Psychologist,

2001;15(3):289-305. https://doi.org/10.1123/tsp.15.3.289

39.Vine S, Wilson M. The influence of quiet eye training and pressure on attention and visuo-motor control. Acta Psychologica, 2011;136(3):340-346. https://doi.org/10.1016/j.actpsy.2010.12.008

40.Vine S, Moore L, Wilson M. Quiet eye training:The acquisition, refinement and resilient performance of targeting skills. EuropeanJournalofSportScience,2014;14(sup1):S235-S242. https://doi.org/10.1080/17461391.2012.683815

41.Vickers JN, Causer J, Vanhooren D. The Role of Quiet Eye Timing and Location in the Basketball Three-Point Shot: A New Research Paradigm. Front Psychol, 2019;10:2424. https://doi.org/10.3389/fpsyg.2019.02424

42.Moeinirad S, Abdoli B, Farsi A, Ahmadi N. The Role of Quiet Eye Duration and Its Components in a Complex Far-Aiming Task. Journal of Motor Learning and Development, 2020;8(3):516-527. https://doi.org/10.1123/jmld.2019-0048

43.Janelle C, Hillman C, Apparies R, Murray N, Meili L, Fallon E et al. Expertise Differences in Cortical Activation and Gaze Behavior during Rifle Shooting. Journal of Sport and Exercise Psychology. 2000;22(2):167-182. https://doi.org/10.1123/jsep.22.2.167

44.Vine S, Wilson M. Quiet Eye Training: The Effects of Physiological Arousal, Cognitive Anxiety, and Gaze Control in Biathlon. Journal of Motor Behavior, 2007;39(5):381-394. https://doi.org/10.3200/JMBR.39.5.381-394

45.Klostermann A. Especial skill vs. quiet eye duration in basketball free throw: Evidence for the inhibition of competing task solutions. European Journal of Sport Science, 2019;19(7):964-971. https://doi.org/10.1080/17461391.2019.1571113

46.Vickers J, Williams A. Performing Under Pressure: The Effects of Physiological Arousal, Cognitive Anxiety, and Gaze Control in Biathlon. Journal of Motor Behavior, 2007;39(5):381-394. https://doi.org/10.3200/JMBR.39.5.381-394

47.Williams A, Vickers J, Rodrigues S. The Effects of Anxiety on Visual Search, Movement Kinematics, and Performance in Table Tennis: A Test of Eysenck and Calvo's Processing Efficiency Theory. Journal of Sport and Exercise Psychology, 2002;24(4):438-455. https://doi.org/10.1123/jsep.24.4.438

\section{Information about the authors:}

Ece Ayaz Kanat; (Corresponding Author); https://orcid.org/0000-0002-1738-1706; eayaz@anadolu.edu.tr; Graduate School of Health Sciences, Eskişehir Anadolu University; İzmir, Turkey.

Deniz Şimşek; Dr., Assoc. Prof.; https://orcid.org/0000-0001-5452-6006'ds@eskisehir.edu.tr; Faculty of Sport Sciences, Eskişehir Technical University; Eskişehir, Turkey.

Cite this article as:

Ayaz Kanat E, Şimşek D. The 'Quiet Eye' and motor performance in basketball free throw shooting. Physical Education of Students, 2021;25(2):103-109.

https://doi.org/10.15561/20755279.2021.0205

This is an Open Access article distributed under the terms of the Creative Commons Attribution License, which permits unrestricted use, distribution, and reproduction in any medium, provided the original work is properly cited http://creativecommons.org/licenses/by/4.0/deed.en

Received: 23.01.2021

Accepted: 10.03.2021; Published: 30.04.2021 